\title{
CHEMISTRY
}

\section{Volatile times for ionic liquids}

\author{
Peter Wasserscheid
}

\section{lonic liquids are useful substances, but in certain applications their utility is limited because they are involatile - or so we thought. In fact, some ionic liquids can be distilled, and even thermally separated.}

The unique physical and chemical properties of ionic liquids - molten salts characterized by melting points below $100{ }^{\circ} \mathrm{C}-$ can be used to enhance the efficiency of a wide range of electrochemical, analytical, synthetic and engineering processes'. This has generated huge interest in these liquids, with their first applications in industrial organic synthesis now being reported ${ }^{2,3}$. On page 831 of this issue 4 , Earle and colleagues, a collaboration of researchers from Portugal, Northern Ireland and the United States, report that some ionic liquids are volatile, and so can be distilled. The findings shake up the received wisdom that ionic liquids are involatile as a class, and might extend their range of applications still further.

Exactly how an ionic liquid behaves varies sharply according to its specific ionic composition: for example, both highly hydrophobic and highly hydrophilic liquids can be produced (Fig. 1). Certain ionic liquids are known to undergo thermal decomposition through mechanisms such as the transfer of an alkyl group ${ }^{5}$ or, in the case of protic ionic liquids ${ }^{6}$ (whose cation is a protondonating, or Brønsted, acid), through deprotonation. For some imidazoliumbased ionic liquids, in which a weakly acidic proton is bound to a carbon atom, the formation of highly reactive organic molecules known as carbenes in the presence of a strong base has also been reported ${ }^{7,8}$. The decomposition products are usually volatile, and, in some cases, reformation of an ionic liquid in colder parts of the apparatus has been observed.

Involatility had been assumed to be a property common to all ionic liquids that do not undergo thermal decomposition. Earle and colleagues ${ }^{4}$ change that assumption, showing that certain ionic-liquid structures known for their very high thermal stability, in particular bis\{(trifluoromethyl)sulphonyl\}amide salts, can be evaporated and recondensed under relatively mild conditions. The distillation of 1-hexyl-3-methylimidazolium bis\{(trifluoromethyl)-sulphonyl\}amide, for instance, is reported to occur at $170^{\circ} \mathrm{C}$ and at a pressure of 0.07 millibar.

It should be noted that Earle and colleagues do not give direct proof for the presence of ions in the gas phase of their distillation



Figure 1| A class apart. Ionic liquids show a range of properties. Here, hydrophobic 1-ethyl-3-methylimidazolium bis\{(trifluoromethyl)-sulphonyl\}amide (bottom phase) is separated from the equally hydrophobic alkane nonane by blue-dyed water. Involatility was, however, assumed to be a characteristic common to all members of the class - until Earle and colleagues ${ }^{4}$ showed otherwise.

processes. But they do describe several instances of ionic liquid evaporation and recondensation for which alkyl-and protontransfer processes leading to neutral volatiles are both highly unlikely. Most interestingly, they report the separation by distillation of a mixture of two ionic liquids. This is also by itself no absolute proof that ions are present in the gas phase: the separation could, in at least one of the demonstrated cases, just reflect the different volatility of carbenes formed in a process of decomposition. Yet despite these reservations, the evidence for the volatility of at least some ionic liquids is convincing.

At first sight, these findings ${ }^{4}$ seem to contradict previous studies that have reported applications of ionic liquids reliant on their blanket non-volatility. Ionic liquids have, for instance, been used in extractive distillation processes, and tested ${ }^{9}$ for their long-term stability over three months at $175^{\circ} \mathrm{C}$ and 50 millibar. Those tests showed no significant loss of the ionic liquid. Ionic liquids have also been used in ultra-high vacuum conditions of $10^{-9}$ millibar for X-ray photoelectron spectroscopy analysis without decomposing or evaporating ${ }^{10}$.

Detailed analysis, however, reveals that the ionic liquids and reaction conditions used in these previous studies differ significantly from those used by Earle and colleagues. The clear corollary of the authors' findings is that non-volatility is not a property that can be assumed for all thermally stable ionic liquids: vapour pressure is just another physical-chemical property that depends on an ionic liquid's cation- 8 anion combination, and will in the future have to be checked experimentally for each new liquid. Adjusting the vapour pressure of the ionic liquid will become an integral part of finding the best ionic liquid for a given application.

Do these developments bring into question the view that ionic liquids are 'green solvents'? That reputation is built largely on their non-volatility, because they do not create the atmospheric pollution that can result from the volatility of classic organic solvents. The answer is no: Earle and colleagues clearly state that the vapour pressure of ionic liquids remains negligible at near ambient conditions, and many ionic liquids show no signs of distillation below the temperature of their thermal decomposition. What the proven volatility of some thermally stable ionic liquids under relatively mild (and close to technically realistic) conditions will most assuredly $\mathrm{do}$, however, is make these substances more useful for us. New purification methods and reactions that use ionic liquids in the gas phase are territories that are now ripe to be explored. The possibility of separating two ionic liquids by distillation will, in particular, unlock the door to new routes towards the manufacture of ionic liquids, and towards the regeneration of spent ionic liquids from technical processes.

Peter Wasserscheid is in the Department of Chemical Reaction Engineering, University of Erlangen, Egerlandstraße 3,91058 Erlangen, Germany.

e-mail:wasserscheid@crt.cbi.uni-erlangen.de

1. Wasserscheid, P.\& Welton, T. (eds) Ionic Liquids in Synthesis (Wiley-VCH. Weinheim 2003).

2. Freemantle, M. Chem. Eng. News 81,9 (2008).

3. Mase, M. in Multiphase Hamageneous Catalysis (eds Comils, B. et a.) 560-566 (Wiley-VCH, Weinheim, 2005).

4. Earle, M.J.etal. Nature 439, 83:-834 (2006).

5. Mase, M. World patent Wo 05/068404 (2005).

6 Volland,M.etal.World patent W003/062251(2003)

7. Earle, M.J.\&. Seddon, K.R. World patent WO O1/077081 (200i).

8. Maase, M.\& Massonne, K. World patent wo $05 / 019183$ (2005).

9. Beste, $Y$, Eggersmann, M. \& Schoenmakers $H$. Chem. ing. Tech 77, 1800-1808 (2005).

10. Smith E F, Villar Garcia, L. L, Briggs, D. \& Licence P. Chem. Commun. 5633-5635 (2005). 